\title{
Wer „ernährt" wen? Auf der Suche nach einem neuen Leitbild
}

\author{
CHRISTINA KLENNER
}

\section{Abschied vom männlichen Familien- ernährer}

Wir erleben derzeit in allen Industriestaaten einen fundamentalen, vermutlich unumkehrbaren Wandel: Das normativ leitende und faktisch weit verbreitete männliche Familienernährermodell verliert zusehends an Boden, die Frauenerwerbstätigkeit ist stark angestiegen, die Lebensformen haben sich pluralisiert, Geschlechterarrangements auf der Paarebene wandeln sich hin zur Zweiverdienerfamilie, Kinder werden zunehmend öffentlich betreut. Vieles steht im Einklang mit dem Modell, das von der Europäischen Union (EU) vor gut zehn Jahren als neues Leitbild formuliert wurde: das „adult worker“-Modell (Modell des „erwachsenen Erwerbsbürgers“). Es ist Teil einer umfassenden Arbeitsmarktaktivierungsstrategie: Jede und jeder Erwachsene soll erwerbstätig sein. Es beinhaltet zugleich, dass Frauen ihren Lebensunterhalt nicht länger abhängig von einem Mann erhalten, sondern postuliert die eigenständige Existenzsicherung für jede Frau ebenso wie für jeden Mann.

Das führt zu der Frage, ob der Familienlohn noch zeitgemäß ist. Der Familienlohn als Leitbild ${ }^{\bullet}$ im fordistischen Industriezeitalter sollte es dem Arbeiter ermöglichen, mit seinem Lohn eine Familie - Frau und Kinder - zu unterhalten. Dieses Bild korrespondiert mit einer Familienform, die heute längst nicht mehr die alleinige oder auch nur typische ist. Und es passt zu einer Vorstellung, die die Abhängigkeit der Frau vom Mann und somit eine hierarchische Geschlechterbeziehung akzeptiert. Die Mehrheit der Menschen in Deutschland hat inzwischen andere Geschlechterrollenvorstellungen. Der Familienlohn ist damit zum einen Ausdruck eines Geschlechtermodells, das heute aus der Gleichstellungsperspektive zunehmend infrage gestellt wird.

Zum anderen hat der Familienlohn heute auch faktisch stark an Prägekraft eingebüßt. Selbst männliche Normalarbeitnehmer - in Vollzeitarbeit, berufsfachlich qualifiziert und nicht atypisch beschäftigt - erzielen heute nur noch zum kleineren Teil Familienlöhne im traditionellen Sinne. Das zeigen die Analysen in den Beiträgen dieses Heftes (vgl. Gottschall/Schröder, Schröder/Schäfer und Berninger/Dingeldey). Frauen, die zunehmend im Dienstleistungssektor erwerbstätig wurden, erzielen selbst auch im Normalarbeitsverhältnis selten einen Familienlohn.

\section{Wir sind mitten im Übergang}

Das von der EU propagierte Modell der „erwachsenen Erwerbsbürger" mit einer eigenständigen Existenzsicherung für alle Menschen ist noch lange nicht Realität, aber die Erosion des männlichen Familienernährermodells vollzieht sich bereits seit Längerem. $\mathrm{Zu}$ welchen Erwerbs- und Geschlechtermodellen der gegenwärtige Übergang führt, ist noch nicht scharf konturiert. Ob Frauen und Männern das ,,adult worker“-Modell tatsächlich durchgängig leben werden, oder ob sie in ihrem Handeln etwas Neues schaffen, ist noch unklar. Wir befinden uns quasi in einem „Übergangsfeld“: Das Alte gilt nicht mehr unangefochten, das Neue ist noch verschwommen. Um neue Leitbilder wird gerungen.

Die Entwicklung der letzten 20 bis 30 Jahre ist im Westen (im Osten rund eine Generation früher) davon gekennzeichnet, dass Frauen in immer größerer Zahl erwerbstätig wurden, allerdings zunächst oft nur in geringem Umfang. Die
Kombination „Mann Vollzeit - Frau Teilzeit“ macht in Deutschland an allen Erwerbskonstellationen in Paarhaushalten mit 35 \% (bei Paaren mit Kindern $45 \%$ ) den größten Anteil aus. ${ }^{2}$ Dieses „modernisierte männliche Familienernährermodell“ beseitigt nicht die Asymmetrie in den Geschlechterarrangements: Frauen schultern weiterhin die Hauptlast der unbezahlten Hausund Sorgearbeit, Männer bleiben die Versorger im finanziellen Sinne. Die Frau erwirbt im modernisierten Familienernährermodell nur einen bescheidenen Hinzuverdienst. Frauen können kurze Arbeitszeiten und niedrige Verdienste aufgrund des Haushaltszusammenhangs akzeptieren, ohne in Armut zu leben, weil und insoweit sie vom auskömmlichen Einkommen des Mannes mit versorgt werden. Doch dass die Zuschreibung und Selbstzuschreibung, die Frau als bloße „Hinzuverdienerin“ anzusehen, verheerend auf die Frauenlöhne wirkt, darauf haben Frauenrechtlerinnen schon vor mehr als 100 Jahren hingewiesen: Aus der untergeordneten Stellung der Hausfrau und ihrer nicht bezahlten Arbeit folgte eine ,geringere Einschätzung ihrer Arbeitskraft“ ${ }^{\circledR}$ aus der Vorstellung, dass sie nur einen „Zuschuss zum Familieneinkommen “ erwerben, niedrigere Lohnforderungen. „Mit diesen niedrigeren Lohnforderungen schaden sich aber nicht nur die Frauen, die nicht aufs Verdienen angewiesen sind, selbst; sondern sie drücken die Löhne ihrer Kon-

\footnotetext{
In der Realität erzielten nie alle Männer einen solchen Familienernährerlohn. Der Lohn hat vie le Bestimmungsfaktoren und gerade Arbeiterfamilien konnten sich oft nicht nur auf ein Einkommen stützen. Doch in der zweiten Hälfte des 20. Jahrhunderts wurde es durch Lohnsteigerungen in industriellen Kernsektoren in einigen westlichen Industrieländern für größere Teile der Arbeiterschaft möglich, das (zunächst bürgerliche) Ideal der Sphärentrennung - der Mann als Familienernährer, die Frau als Familienversorgerin - tatsächlich zu leben (vgl. Crompton, R. (ed.) (1999): Restructuring gender relations and employment. The decline of the male breadwinner,
}

Oxford/New York). Eine massive staatliche Stützung dieses Modells gerade in Deutschland tat ein Übriges, es zu ermöglichen und zu festigen.

(2) Vgl. Brehmer, W./Klenner, Ch./Klammer, U. (2010): Wenn Frauen das Geld verdienen - eine empirische Annäherung an das Phänomen der „Familienernährerin“, WSI Diskussionspapier (170), Düsseldorf.

(3) Salomon, A.: Gleicher Lohn für gleiche Leistung (1906), in: Brinker-Gabler, G. (Hrsg.) (1979): Frauenarbeit und Beruf. Die Frau in der Gesellschaft. FrüheTexte, Frankfurt a. M., S. 197. 
kurrentinnen, die von der Arbeit leben müssen, mit herunter. ${ }^{\text {“ब }}$

Dass Frauen auch heute im Durchschnitt deutlich, rund ein Viertel, pro Stunde weniger verdienen, ist auch der nach wie vor geringeren Bewertung weiblich dominierter Berufe geschuldet. Hinter dieser dürfte auch die Konstruktion des weiblichen Einkommens als „Hinzuverdienst“ stehen.

\section{Nichtexistenzsichernde Individual- einkommen}

Mag der Haushaltszusammenhang Armut verhindern, so sind doch die individuellen Verdienste von zahlreichen Erwerbstätigen, vor allem Frauen, oftmals nicht existenzsichernd. Das gilt für die Einkommen der rund 4,9 Mio. Arbeitnehmerinnen und Arbeitnehmer, zu zwei Dritteln Frauen, die einen Minijob als einzige Erwerbstätigkeit ausüben. ${ }^{\ominus}$ Das trifft darüber hinaus aber für vie le weitere Niedriglohnbeschäftigte zu. Die Analysen in diesem Heft zeigen, dass auch beruflich qualifizierte Frauen im Normalarbeitsverhältnis davon betroffen sind. ${ }^{\circledR}$ Insgesamt erzielt rund ein Drittel der abhängig beschäftigten Frauen in Deutschland derzeit kein existenzsicherndes Einkommen für sich selbst; rechnet man den Lebensunterhalt für ein Kind dazu, ist es fast jede zweite Frau (45\%). ${ }^{\ominus}$ Wenn darüber hinaus in einer Lebenslaufperspektive die Existenzsicherung bis ins Rentenalter hinein zur Grundlage genommen wird, dann ist diese sogar für $62 \%$ der Frauen aus ihrem aktuellen Einkommen nicht gesichert.

Nicht existenzsichernd entlohnt zu werden dieses Schicksal teilen heute in zunehmender Zahl auch Männer. 15 \% der Männer erzielen derzeit keinen existenzsichernden Lohn; bezieht man die Existenzsicherung zusätzlich auf ein Kind, dann verdienen $29 \%$ der Männer nicht genug, um sich und das Kind finanziell zu unterhalten. ${ }^{8}$
Für Bezieher und Bezieherinnen niedriger individueller Erwerbseinkommen spielt also der Haushaltszusammenhang zur Armutsvermeidung eine zentrale Rolle. ${ }^{\ominus}$ Das gilt auch für viele der "Normalarbeitnehmer", wie Berninger/ Dingeldey in diesem Heft zeigen. Viele Erwerbstätigkeiten mit Niedrigeinkommen könnten gar nicht akzeptiert werden, würden die Niedrigein kommenbezieher und -bezieherinnen nicht im Rahmen der Partnerschaft mit versorgt. Ohne das Fortbestehen der Tradition innerfamilialer Versorgungsbeziehungen hätte die Ausbreitung des Niedriglohnsektors zu einem viel deutlicheren Anstieg sozialstaatlicher Transferleistungen oder zu mehr Armutslagen geführt.

\section{Ausdifferenzierte Konstellationen auf Haushaltsebene}

Doch die Mitversorgung einer Partnerin oder eines Partners stößt an Grenzen. Prozesse der Prekarisierung der Erwerbsarbeit, die auch Männer treffen können, haben einen Teil von ihnen der Chance beraubt, eine Familie zu versorgen. Längst ist nun in Deutschland der männliche Familienernährer nicht mehr selbstverständlich. ${ }^{(1)}$

Im Gegenzug ist ein Teil der Frauen zu Familienernährerinnen geworden. Dies gilt aktuell für $11 \%$ der Paarhaushalte. ${ }^{\mathbb{1}}$ Zusammen mit den $\mathrm{Al}$ leinerziehenden, die auch finanziell für ihre Kinder sorgen, erwirtschaften Frauen somit in rund jedem fünften Mehrpersonenhaushalt hauptsächlich oder allein die finanzielle Existenzgrundlage. In Ostdeutschland wird bereits jeder vierte Mehrpersonenhaushalt hauptsächlich von einer Frau versorgt Dabei hat nur ein kleiner Teil der Frauen infolge einer Absprache mit dem Partner bewusst die Rolle der Familienernährerin übernommen. Oft geraten die Frauen unvorbereitet in diese Verantwortung hinein, weil ihre Männer arbeitsmarktbedingt die Familie nicht mehr ernähren können.
Familienernährerinnen erhalten oft kein Einkommen, von dem sie eine Familie oberhalb der Armutsschwelle oder mit einem mittleren Lebensstandard versorgen könnten. $30 \%$ der Familienernährerinnen verdienen weniger als $900 €$ individuelles Erwerbseinkommen; auch für $4 \%$ der männlichen Familienernährer trifft das zu. ${ }^{(2)}$

Auf der anderen Seite gibt es einen wachsenden Teil der Paare, bei denen beide in etwa gleich viel zum Familieneinkommen beitragen. Dies sind in Deutschland $30 \%$, im Osten sogar $44 \%$ der Paarhaushalte.

Empirisch zeigt sich also ein ausdifferenziertes Bild, in dem sich die Geschlechtergrenzen teilweise verwischt haben: Niedrige Löhne sind nicht mehr auf hinzuverdienende Frauen beschränkt, Männer als Familienernährer sind nicht mehr die Regel. Auch Frauen versorgen Familien als Familienernährerinnen, ein Teil von ihnen muss das aber, ebenso wie ein Teil der Männer, ohne einen ausreichenden Familienlohn tun, sodass diese Familien nur in Armut leben können.

\section{Auf der Suche nach einem neuen Leitbild}

Ausgangspunkt einer Debatte um existenzsichernde Einkommen, aus denen Frauen und Männer, ihre Kinder und ggf. ihre Partner versorgen können, muss die Vielfalt unterschiedlicher Lebensformen sowie Erwerbs- und Einkommenskonstellationen sein. In fast einem Drittel der Haushalte ${ }^{\circledR}$ wirtschaftet ein Mann oder eine Frau für sich allein in weiteren $7 \%$ Alleinerziehenden-Haushalten gibt es ebenfalls nur eine Erwerbsperson. Für diese $38 \%$ aller Erwerbshaushalte stellt sich das Problem zwar nicht, weitere erwachsene Haushaltsmitglieder ökonomisch versorgen zu müssen, es ist aber keineswegs auszuschließen, dass sie ehemalige Partner oder nicht bei ihnen lebende Kin der zu finanzieren haben. Daneben gibt es
(4) Ebd. (Hervorhebung der Autorin).

5 Weinkopf, C. (2012): Stellungnahme zur öffentlichen Anhörung des Ausschusses für Arbeit und Soziales im Deutschen Bundestag am 22. Oktober 2012, Daten der Bundesagentur für Arbeit 2012.

6 Vgl. Berninger/Dingeldey in diesem Heft.

(7) Pimminger, I. (2012): Existenzsichernde Beschäftigung von Frauen und Männern, Online verfügbar unter http://www.esf-gleichstellung.de/ fileadmin/data/Downloads/Aktuelles/expertise existenzsichernde_beschaeftigung.pdf, zuletzt aktualisiert am 08.10.2012 (Letzter Zugriff: 06.02.2013).
8 Ebd., S. $31 f$.

(9) Göbel, J./Krause, P./Schupp, J.: Mehr Armut durch steigende Arbeitslosigkeit. Niedriglöhne überwiegend als Zusatzeinkommen im Haushalt, DIW Wochenbericht 72 (10), Berlin.

(10) Dennoch überwiegt noch die Zahl der Familienhaushalte, in denen der Mann den Hauptteil des Einkommens verdient. 2010 war das in knapp $60 \%$ der heterosexuellen Paarhaushalte der Fall. Die Daten wurden im Auftrag des WSI von Tanja Schmidt, Sozialforschung Berlin, berechnet. Eine gemeinsame Publikation zu Bestimmungsfaktoren der Einkommensrelationen in Paarhaushalten ist in Vorbereitung.
(11) Datenquelle vgl. Fußnote 10. Die Zahl bezieht sich auf heterosexuelle Paare. Da unsere Forschungen auf die Veränderung von Geschlechterarrangements fokussiert waren, haben wir gleichgeschlechtliche Paarhaushalte aus der Betrachtung ausgeklammert. Vgl. auch Klenner, Ch./Menke, K./ Pfahl, S. (2012): Flexible Familienernährerinnen. Moderne Geschlechterarrangements oder prekäre Konstellationen?, Opladen et al.

(12) Angaben für 2007; http://www.boeckler. de/38555 38694.htm.

(13) Haushalte mit einer Erwerbsperson unter 65 Jahren; vgl. Brehmer, W./Klenner, Ch./Klammer, U. (2010), a.a.O 
heterosexuelle und gleichgeschlechtliche Paarhaushalte mit einem oder zwei Verdienern, mit Vollzeit- oder Teilzeitbeschäftigung eines Partners oder beider Partner. Der Familienlohn traditionellen Zuschnittes als Leitvorstellung für einen Mann als Familienernährer mit nicht erwerbstätiger Ehefrau findet seine Entsprechung in der Realität der Lebensformen heute also nur noch zu einem kleinen Teil.

Daher stellen sich mit den gegenwärtigen Umbrüchen in der Erwerbswelt, in den Geschlechterverhältnissen und in der sozialen Sicherung viele normative Fragen neu. Wie sollen Familien künftig leben, wer soll für wen sorgen, wer zahlt für die Kinder?

Wenn der Familienlohn für den männlichen Familienernährer immer weniger zur Realität passt, benötigen dann nicht aber vielleicht Männer und Frauen einen Familienlohn neuen $\mathrm{Zu}$ schnitts? Oder ist es besser, von eigenständiger Existenzsicherung aller Frauen und Männer zu sprechen - wobei dann zu klären wäre, was darunter zu verstehen ist? Wie viele Kinder müssten von einem Erwachsenen mit versorgt werden können? Und gehört die zeitweilige Versorgung des Partners oder der Partnerin zu einem künftigen Leitbild, müsste eine eigenständige Existenzsicherung mithin auch dies ermöglichen?

In der deutschen Mindestlohndebatte wird vorwiegend vom Lebensunterhalt (und der Alterssicherung) für einen einzelnen Menschen ausgegangen. ${ }^{(0)}$ Pimminger unterstellt in ihren Modellrechnungen für existenzsichernde Einkommen neben der individuellen Absicherung den Unterhalt für ein Kind. ${ }^{\oplus}$

Welcher Einkommensmix soll in Zukunft für verschiedene Lebenslagen und Haushaltskonstellationen angestrebt werden? Derzeit ist es so, dass das Erwerbseinkommen die Hauptquelle des Lebensunterhaltes ist, dass aber viele Bezieherinnen und Bezieher unterer Einkommen auf Partnereinkommen oder aufstockende Transferleistungen des Staates zur Existenzsicherung angewiesen

(44) Vgl. etwa Schulten,Th. (2009): Guter Lohn für gute Rente, WSI-Diskussionspapier (164), Düsseldorf

(15) Vgl. Pimminger, a.a.O.

(16 Vgl. zum Beispiel "Der 200-Milliarden-Irrtum”, in: Der Spiegel 6/2013, S. 22-29.

(17) Lewis, J. (2004): Auf dem Weg zur „ZweiErwerbstätigen“-Familie, in: Leitner, S./Ostner, I./ Schratzenstaller, M. (Hrsg.): Wohlfahrtsstaat und Geschlechterverhältnis im Umbruch. Was kommt nach dem Ernährermodell? Wiesbaden, S. 62-84. sind. Wie soll dieser Mix künftig aussehen? Was soll der Sozialstaat fördern und finanzieren?

Ob Familienlohn oder eigenständige Existenzsicherung: das neue Leitbild ist demokratisch auszuhandeln. Und die Rahmenbedingungen dafür sind politisch zu gestalten. In diesem Suchprozess geht es unter anderem um folgende Fragen:

- Wer zahlt für den Lebensunterhalt und die Bildung der Kinder? In welcher Kombination kommen die Eltern, der Staat oder evtl. Sozialversicherungen für sie auf?

- Wer sichert den Lebensunterhalt unfreiwillig nicht erwerbstätiger, arbeitsloser, erwerbsunfähiger Menschen? Inwieweit sollen und können Partner im Haushalt dafür herangezogen werden? Oder sollen Arbeitslose und Erwerbsunfähige vollständig vom Staat oder von der Versichertengemeinschaft finanziert werden?

- Soll die Subventionierung des Unterhalts freiwillig nichterwerbstätiger Partner fortgesetzt werden? Heute werden Ehen in hohen und mittleren Einkommenslagen über das Ehegattensplitting und die kostenfreie Mitversicherung in der Krankenkasse subventioniert.

- Wie hoch muss ein Erwerbseinkommen sein, damit auch in der Nacherwerbsphase eine auskömmliche Rente bezogen werden kann?

- Wie lässt sich ein existenzsicherndes Einkommen in derzeit frauendominierten Niedriglohnbranchen durchsetzen?

Wie aktuelle Debatten zeigen, ist der Streit um die „richtigen“ Leitbilder voll entbrannt. ${ }^{\circledR}$ Dabei stellen sich auch Gerechtigkeitsfragen: Welche Ehen profitieren zum Beispiel vom Ehegattensplitting und welche nicht? Wer muss im Rahmen der Bedarfsgemeinschaften andere mitversorgen und rutscht dadurch womöglich selbst in Armutsnähe?

Schließlich ist das Ziel - der „erwachsene Erwerbsbürger" - selbst wohl noch einmal nachzujustieren. Soll es wirklich das künftige Leitbild sein, dass alle von der Ausbildung bis zur Rente

(8) Gornick, J. C./Meyers, M. K. (2005): Supporting a Dual Earner/Dual-Carer Society, in: Heymann, J./Beem, Ch. (Hrsg.): Unfinished work. Building equality and democracy in an era of working families, New York, S. 371-408.

(1) Krüger, H. (2006): Geschlechterrollen im Wandel - Modernisierung der Familienpolitik, in: Bertram, H./Krüger, H./Spiess, K. (Hrsg.): Wem gehört die Familie der Zukunft? Expertisen zum 7. Familienbericht der Bundesregierung, Opladen, S. 191-206. in Vollzeit erwerbstätig sein sollen und müssen, um ihre Existenz zu sichern? Welche Tätigkeiten rechtfertigen in Zukunft eine sozialstaatlich unterstützte Auszeit vom Beruf, wie sie derzeit (fast nur) mit dem Elterngeld möglich ist?

\section{„Dual earner - dual carer“ statt „adult worker"-Modell}

Das propagierte „adult worker“-Modell lässt die Frage der Sorgearbeit weitgehend außer Acht. ${ }^{(0)}$ Nötig erscheint daher, eine gesellschaftliche Debatte über künftige Formen der Verbindung von Einkommenserwerb und Fürsorge im Lebensverlauf zu führen. Der Bereich der Sorgeverantwortung ist in all seinen Facetten in der Debatte um künftige Modelle von Arbeiten und Leben auszuleuchten. Mit der Versorgung von Kleinkindern in öffentlichen Einrichtungen, selbst wenn diese flächendeckend erreicht wäre, ist es dabei keinesfalls getan. Es geht auch um ältere Kinder, um Pflege, um Selbstsorge und vieles mehr.

Zukunftsweisend und geschlechtergerecht könnte ein Modell („dual earner - dual carer“Modell) ${ }^{\circledR}$ sein, bei dem beide, Mann und Frau, arbeitsmarktaktiv und familienaktiv sein können. ${ }^{\circledR}$ Das heißt, dass alle Menschen unabhängig vom Geschlecht Geld erwerben und fürsorglich tätig sein können und dass auch in verschiedenen Lebensphasen der Umfang der Erwerbstätigkeit und der Fürsorgetätigkeit wie auch anderer Aktivitätsformen variieren kann, ohne dass die Existenzsicherung infrage gestellt ist.

$\mathrm{Zu}$ einem Familienlohn neuartigen $\mathrm{Zu}$ schnitts gehören nicht nur die individuelle Existenzsicherung sowie die finanziellen wie zeitlichen Möglichkeiten, um Kinder versorgen zu können. Dazu gehört auch und nicht zuletzt, die typischerweise von Frauen geleistete Erwerbsarbeit entsprechend der Qualifikation, Anforderungen und Beanspruchungen adäquat zu entlohnen und die implizite Bezugnahme von Fraueneinkommen auf einen „Hinzuverdienst“ zu beenden. Die Durchsetzung einer gleichen Bezahlung für gleiche und gleichwertige Arbeit über Branchen hinweg ist eine dringende, gesamtgesellschaftlich zu lösende Aufgabe.

\section{AUTORIN}

CHRISTINA KLENNER, Dr., ist Wissenschaftlerin im Wirtschafts- und Sozialwissenschaftlichen Institut (WSI) der Hans-Böckler-Stiftung. Arbeitsschwerpunkte: Geschlechterverhältnisse, Frauenerwerbstätigkeit und Work-Life-Balance, Gleichstellung.

christina-klenner@boeckler.de 